\title{
POTASSIUM HUMATE REDUCES INFLAMMATION AND CLINICALLY IMPROVES THE OUTCOMES OF PATIENTS WITH OSTEOARTHRITIS OF THE KNEE.
}

\author{
CONSTANCE E. VAN RENSBURG*, BREGGIE E. \\ BADENHORST, JUSTIN J. GANDY, JACQUES R. SNYMAN.
}

\author{
Department of Pharmacology, Faculty of Health Sciences, \\ University of Pretoria, Pretoria, South Africa
}

Corresponding author: connie.medlen@up.ac.za

Key words: potassium humate; inflammation; osteoarthritis of the knee

\begin{abstract}
A pilot study was done to determine if potassium humate, a natural substance derived from brown coal, with known anti-inflammatory properties, is safe and effective in reducing pain and inflammation in osteoarthritis of the knee. This was conducted as a randomized, double-blind, placebo-controlled, single centre, cross-over. Participants were enrolled for a total of 14 weeks, starting with an initial 1-week washout period, after which they were randomly assigned to either potassium humate or lactose, administered orally for 6 weeks at a dosage of $600 \mathrm{mg}$ three times daily. Following another 1-week washout period, participants were crossed over to the other treatment for another 6 weeks. Participants were not permitted the use of anti-inflammatory medications. Paracetamol was allowed as rescue medication for the duration of the trial. The primary efficacy variable were the WOMACтM scores (visual analogue version) for pain, stiffness, physical function and total score and health related issues using the RAND 36 levels, rescue medication use, adverse effects and tolerability.

28 participants were enrolled and 21 participants successfully completed the protocol. A carry-over effect in the stiffness subscale was observed. There was a significantly greater clinical benefit with potassium humate over placebo with reduction in all the WOMAC subscale scores for pain. After adjusting for baseline, potassium humate showed a greater reduction in hs-CRP levels when compared to placebo. Tolerability was good for all groups. Safety parameters remained unchanged, except for an increase in the GGT-levels $(n=4$ in potassium humate group, $\mathrm{n}=2$ in the placebo group). Levels of GGT returned to baseline within 2 weeks of discontinuation of therapy. In conclusion, potassium humate showed benefit over placebo in patients with OA of the knee, with a statistically significant reduction in hs-CRP levels. The patient reported outcomes proved a clinically significant benefit of potassium humate over placebo. The small sample size and the carry-over effect limited further interpretation of data.
\end{abstract}




\section{INTRODUCTION}

Osteoarthritis is one of the most common forms of arthritis, and is a progressive, debilitating disease that affects mainly elderly people [1]. It remains a difficult disorder to treat as there is no cure, and current regimes to treat pain and maintain joint function, all have long-term risks for the patients. Paracetamol is not an antiinflammatory drug, but is however regarded as a safe and effective analgesic with a favourable side effect profile, as it causes less gastrointestinal and renal side effects than NSAIDs (non-steroidal anti-inflammatory drugs). It has however demonstrated less symptomatic efficacy than NSAIDs in some clinical trials [2] whereas NSAIDs provide relief of pain and inflammation in osteoarthritis. Both paracetamol and NSAIDs are also associated with adverse effects, such as gastrointestinal toxicity, renal function impairment [3] and increased cardiovascular risk [4, 5].

Inflammation is increasingly recognized as a factor contributing to the symptoms and progression of osteoarthritis $(\mathrm{OA})$, even in the absence of acute inflammatory flares [2]. Inflammatory infiltrates can be found in the synovial membrane of osteoarthritic joints, and there is serological and histological evidence of synovitis, even though osteoarthritis is not associated with specific immune markers [6].

High sensitivity $\mathrm{C}$ reactive protein (hs $\mathrm{CRP}$ ) is a sensitive marker of low grade systemic inflammation and serum levels of CRP correlates well with CRP levels in the synovial fluid of patients with osteoarthritis and rheumatoid arthritis. Increased synovial turnover markers are associated with increased WOMAC ${ }^{\mathrm{TM}}$ scores as well as increased serum CRP [7].

Humic acids are formed during the decomposition of organic matter and can therefore be found in practically all natural environments in which organic materials and micro-organisms are, or have been present [8]. Peat extracts have been used in therapeutic baths for the treatment of rheumatic conditions during the $19^{\text {th }}$ century [9]. It was shown in two recent studies that potassium humate, derived from brown coal, suppresses (i) ear swelling in a contact hypersensitivity animal model, (ii) an increase in paw volume of carrageenan-induced oedema in rats and (iii) a graft-versus-host reaction induced in normal and immune incompetent rats $[10,11]$. It was also found that this product had no effects on the safety parameters tested at $1000 \mathrm{mg} / \mathrm{kg}$ body weight per day when administered to rats by gavage for one month, nor did $500 \mathrm{mg} / \mathrm{kg}$ body weight have any effect on pups 
after oral administration of the product to pregnant female rats, indicating the safety profile of this compound.

The aim of this research was to establish the safety and efficacy (using clinical and laboratory markers) of potassium humate in a pilot study in patients suffering from osteoarthritis of the knee.

\section{MATERIALS AND METHODS}

\section{Study design}

In this randomized, double-blind, placebo-controlled, cross-over single-centre clinical trial [Fig (1)], volunteers were enrolled, after signing an formed consent, for a 14 week study period. This study was reviewed and approved by the Research Ethics Committee of the Faculty of Health Sciences, University of Pretoria. The study started with a 7-day washout period of all anti-inflammatory agents, followed by treatment with either potassium humate or placebo, in a randomized order in two successive treatment periods of six weeks each, separated by another 7-day washout period. Participants were not permitted the use of anti-inflammatory medications such as NSAIDs, cartilage supplements, steroids, or any other agents that may affect the outcomes of the study other than the rescue medication. Any medication taken by the subjects for two months prior to the inclusion of the study, and whose intake was stabilized, was permitted and monitored. The dosing of these medications was not changed for the duration of the investigation.
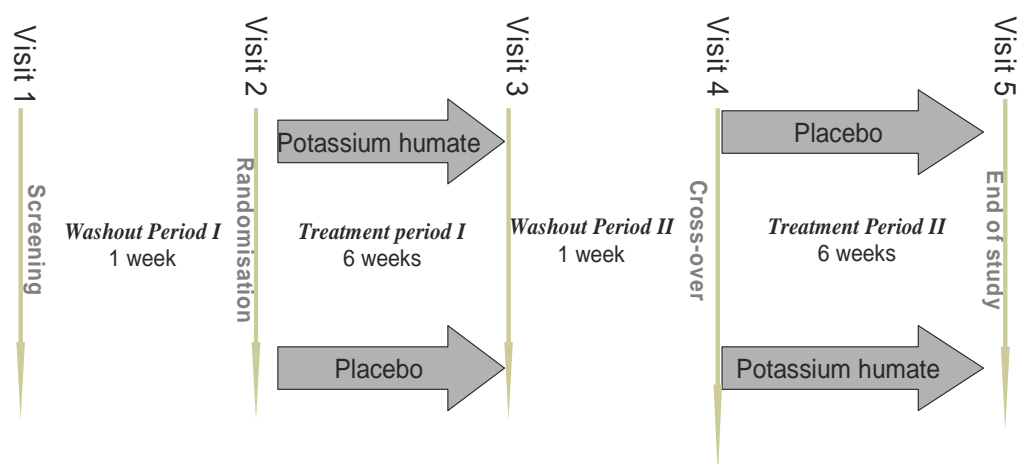

Fig.(1) The trial was done in a cross-over fashion. Participants were enrolled for 14 weeks, starting with a 7-day washout period of all anti-inflammatory agents, followed by treatment with either potassium humate or placebo, in two successive treatment periods of six weeks each, separated by another 7-day washout period.

Paracetamol and tramadol were provided as rescue medication in the first washout period, and paracetamol for the rest of the 
duration of the trial. Participants completed medication diaries to indicate the use of all concomitant and rescue medication.

Screening included clinical assessment, radiographic assessment, laboratory tests and the disease-specific questionnaire, WOMAC ${ }^{\text {TM }}$ (Western Ontario and McMaster Universities Osteoarthritis Index questionnaire) [12] as well as the RAND 36-Item Health Survey [13]. Clinical assessment consisted of an interview and examination by a clinician, and the supervised self-administration of the questionnaire, and a visual analogue scale (VAS) for pain to ensure that patients scored at least $40 \mathrm{~mm}$ on a $100 \mathrm{~mm}$ scale for pain. Radiographic assessment was performed in patients who have not had X-rays previous to the trial. It was done using standard antero-posterior weight-bearing X-ray films of the knees to determine the presence of osteophytes.

\section{Study procedure}

After the initial screening visit, the participants had 4 scheduled visits to the investigator (randomization visit, after first 6-week treatment period, after second washout period, when the treatments were crossed over and after the second 6 -week treatment period). Identical maroon capsules contained $600 \mathrm{mg}$ of either potassium humate or lactose was supplied by Unique Health Trust. All subjects took one capsule of either potassium humate or lactose, three times daily, and were allowed paracetamol 2 tablets 4 times daily (a total maximum of 8 tablets daily) as rescue medication. Compliance was assessed by a pill count at each study visit.

The primary efficacy outcome measure were the WOMAC ${ }^{\text {TM }}$ that measured pain stiffness and function as well as the RAND 36-Item Health Survey measured on a $100 \mathrm{~mm}$ scale which was completed at baseline and at the end of each 6-week treatment period. The study was designed to detect a MCID (minimal clinically important difference) of a reduction of $-9.1 \mathrm{~mm}$ to $-7.9 \mathrm{~mm}$ in the $100 \mathrm{~mm}$ visual analogue scale of the WOMACTM subscale scores. Secondary efficacy outcome measures included serum hs-CRP (high sensitivity C-reactive protein) assays, tolerability, compliance, reduced use of rescue medication and adverse event reporting. The hs-CRP values were determined at baseline (after both wash-out periods) and repeated after each 6-week treatment period.

Use of rescue medication, i.e. paracetamol, was documented as a measure of both pain management and efficacy. The amount of rescue medication was only assessed in terms of total use at the conclusion of the study period. Rescue medication use was 
assessed sequentially along with other variables. Safety outcome measures included the recording of vital signs at each visit and laboratory tests (full blood count, sedimentation rate, creatinine and liver function tests, i.e. ALT and GGT, which were done at screening and repeated at the end of each 6-week treatment period.

\section{Patients}

Patients, aged over 45 years of both male and female gender, with unilaterally symptomatic idiopathic OA of the knee were recruited. To be considered for the study, patients had to meet clinical and radiographic enrolment criteria.

The clinical criteria included the American College of Rheumatologist (ACR) Classification Criteria for Idiopathic Osteoarthritis of the Knee [14] and the presence of pre-enrolment ambulatory pain (defined as a score of at least 40units on a 100unit $\mathrm{mm}$ visual analogue scale (VAS) for pain. The radiographic criteria consisted of the presence of radiographic OA (KellgrenLawrence grade 2 or 3 ) $[15,16]$.

Participants were selected according to standardized inclusion and exclusion criteria. Except for OA of the knee patients had to be otherwise healthy. Recent use of non steroidal anti-inflammatory drugs (NSAIDS) and systemic corticosteroids required a 3 month washout period.

\section{Treatments}

Potassium humate (Zymate ${ }^{\mathrm{TM}}$ ) was obtained from Unique Health Trust, Milnerton, South Africa. The product is marketed as a food supplement. Lactose was used as the placebo.

All treatment capsules were uniform in size, colour, bottle filling, labelling, and packaging. Treatments were packaged in maroon gelatine capsules containing $600 \mathrm{mg}$ of potassium humate or lactose and packed in wide mouthed white opaque plastic bottles with screw caps in a clean room.

\section{Statistical methods}

In this study the RAND 36-Item Health Survey as described by Garratt et al [13], as well as the WOMAC score [15] were used as primary outcome.

Differences in WOMAC scores and health survey parameters were used in treatment groups at baseline, 7 weeks, and 14 weeks, and group wise changes between baseline, 7 weeks and 14 weeks. 
Outcome scores were analyzed for variation in effect for Sequence, Treatment, Period and Carryover. Results for the WOMAC score were analysed using an ANOVA and the health survey results were analysed using the Wilcoxon test for paired values. A $p$ value of $<0.05$ was regarded as significant.

Bonferoni test for multiple comparisons was used to compare differences in hs-CRP values, measured at the onset as well as at the end of each treatment period (week 2 and week 7; week 8 and week 15). Statistical significance was taken at the 95\% level $(p<$ $0.05)$. Results are expressed as the mean $\pm \mathrm{SD}$.

All participants lost to follow-up had been accounted for.

\section{RESULTS}

\section{Subject characteristics and study flow}

Of the 55 patients screened, 28 patients met the clinical and radiographic criteria at enrolment and were randomized The characteristics of the subjects at enrolment and randomization are given in Table 1. 21 Participants successfully completed the protocol. A summary of patient disposition can be seen in Fig. (2).

Table 1: Baseline characteristics of patients on trial

\begin{tabular}{|c|l|}
\hline Age (mean in years) & 63.46 \\
& \pm 9.48 \\
\hline Body Mass Index (BMI) (kg/m2) (mean) & $31 \pm 5$. \\
& 13 \\
\hline Gender: Female & $\mathrm{n}=21$ \\
\hline Male & $\mathrm{n}=7$ \\
\hline Knee affected: Right & $\mathrm{n}=16$ \\
\hline Left & $\mathrm{n}=12$ \\
\hline Duration of OA: 1-5 years & $\mathrm{n}=12$ \\
\hline $5-10$ years & $\mathrm{n}=10$ \\
\hline$>10$ years & $\mathrm{n}=6$ \\
\hline Pre-study pain medication: Paracetamol & $\mathrm{n}=1$ \\
\hline NSAID/ high dose aspirin & $\mathrm{n}=7$ \\
\hline NSAID \& paracetamol & $\mathrm{n}=5$ \\
\hline None & $\mathrm{n}=15$ \\
\hline
\end{tabular}

\section{Clinical Response Primary efficacy variable}

The primary efficacy variable was a clinically significant reduction on a $100 \mathrm{~mm}$ visual analogue scale (VAS) in the WOMAC scores for stiffness, global score and mean score in the group with order placebo cross over to potassium humate (Table 2). This was not the case in the group with order potassium humate cross over to placebo (Table 3) indicating a carry-over effect. 
The health survey parameters were tested using a Wilcoxon Rank test to test for significant variances within each group. The only significant difference obtained in the health survey was between the combined placebo groups and the treatment groups regarding physical functioning, where an increase of 12.86 on the VAS scale was documented vs 6.52 for the placebo group (Table 4).

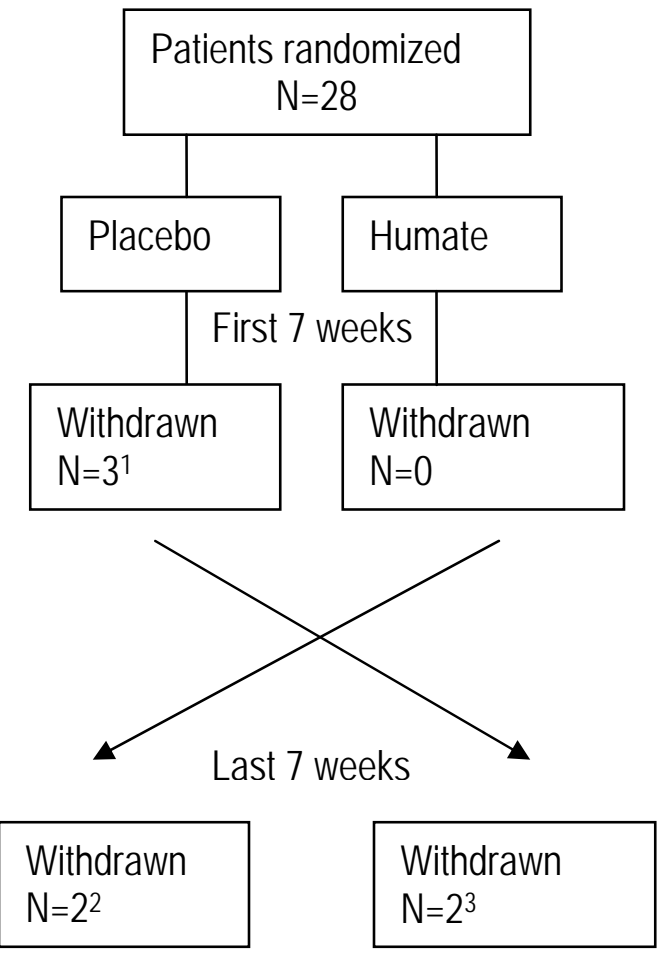

Fig.(2) Patient disposition in the double blind cross-over trial..

${ }^{1}$ One patient withdrawn due to lack of efficacy and two patients due to adverse effects.

${ }^{2}$ One patient withdrawn due to an unrelated adverse event and one patient due to an unrelated reason.

${ }^{3}$ One patient withdrawn due to lack of efficacy and the second patient due to an unrelated reason. 
Table 2. Effect of Potassium humate vs Placebo on the WOMAC ${ }^{\mathrm{TM}}$ scores with respect to baseline and order taken

\begin{tabular}{|c|c|c|c|c|c|c|c|}
\hline & \multicolumn{7}{|c|}{ Visual Analogue Scale $(\min =0, \max =100)$} \\
\hline & \multicolumn{2}{|c|}{ Baseline } & \multicolumn{2}{|c|}{ Placebo } & \multirow{7}{*}{ 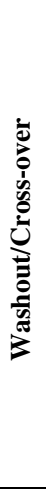 } & \multicolumn{2}{|c|}{ Potassium Humate } \\
\hline & Mean & \pm SD & Mean & \pm SD & & Mean & \pm SD \\
\hline $\begin{array}{l}\text { Pain } \\
\text { Dimension }\end{array}$ & 45.667 & 16.948 & 30.089 & 19.781 & & 29.533 & 26.745 \\
\hline $\begin{array}{l}\text { Stiffness } \\
\text { Dimension }\end{array}$ & 52.333 & 16.145 & 42.611 & 26.726 & & *28.611 & 26.871 \\
\hline $\begin{array}{l}\text { Physical } \\
\text { Function }\end{array}$ & 42.781 & 14.677 & 35.653 & 25.215 & & 27.134 & 26.579 \\
\hline $\begin{array}{l}\text { Global } \\
\text { Score }\end{array}$ & 140.781 & 39.756 & 108.353 & 68.230 & & $* 85.279$ & 79.836 \\
\hline Mean Score & 46.927 & 13.252 & 36.118 & 22.743 & & *28.426 & 26.612 \\
\hline
\end{tabular}

*Indicating statistical significant difference from baseline $(\mathrm{p}<0.05)$

Table3. Effect of Potassium humate vs Placebo on the WOMACтм scores with respect to baseline and order taken

\begin{tabular}{|c|c|c|c|c|c|c|c|}
\hline & \multicolumn{7}{|c|}{ Visual Analogue Scale $(\min =0, \max =100)$} \\
\hline & \multicolumn{2}{|c|}{ Baseline } & \multicolumn{2}{|c|}{ Potassium humate } & \multirow{7}{*}{ 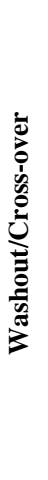 } & \multicolumn{2}{|c|}{ Placebo } \\
\hline & Mean & \pm SD & Mean & \pm SD & & Mean & \pm SD \\
\hline $\begin{array}{l}\text { Pain } \\
\text { Dimension }\end{array}$ & 46.217 & 28.480 & 44.177 & 20.870 & & 35.358 & 23.123 \\
\hline $\begin{array}{l}\text { Stiffness } \\
\text { Dimension }\end{array}$ & 64.000 & 25.733 & 64.892 & 32.479 & & 58.667 & 31.186 \\
\hline $\begin{array}{l}\text { Physical } \\
\text { Function }\end{array}$ & 54.001 & 22.834 & 48.136 & 21.153 & & 46.649 & 27.356 \\
\hline $\begin{array}{l}\text { Global } \\
\text { Score }\end{array}$ & 161.917 & 66.660 & 157.204 & 64.710 & & 140.674 & 78.814 \\
\hline Mean Score & 53.972 & 22.220 & 52.401 & 21.570 & & 46.891 & 26.271 \\
\hline
\end{tabular}

\section{Secondary efficacy variables}

hs-CRP

Potassium humate treated participants, using the combined placebo groups and the treatment group results, had a significant reduction $(\mathrm{p}<0.5)$ in hs-CRP from a mean value of 14.4 to $6.6 \mathrm{mg} / \mathrm{L}$ [Fig. (3)]. 


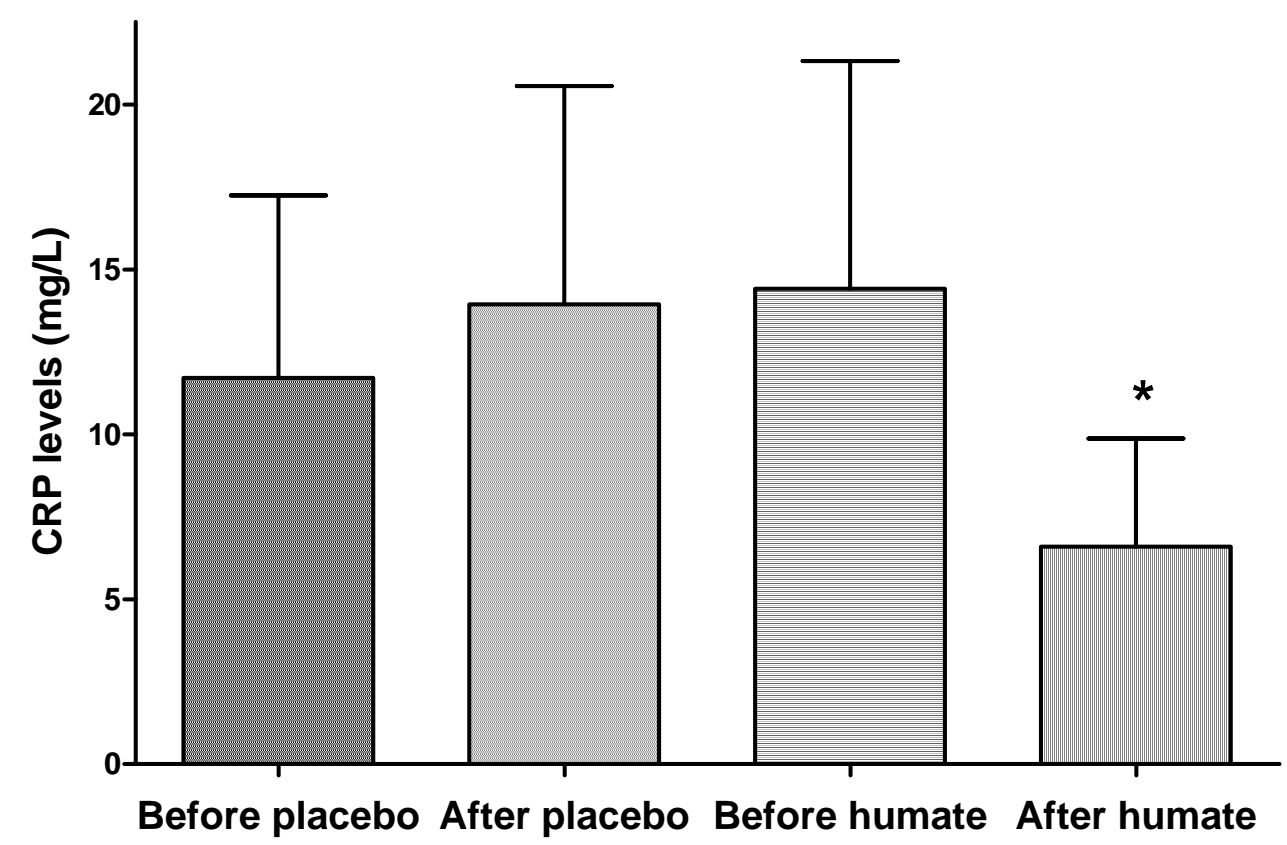

Fig. (3). Comparison of the Potassium Humate vs Placebo on CRP levels.

* Indicating significant difference $(\mathrm{p}<0.05)$ compared to the value before potassium humate treatment.

Patient disposition and consumption of rescue medication and There were no significant differences in patient disposition or the use of paracetamol in both groups.

Tolerability.

Tolerability was good for all groups. Pill counts performed at each study visit demonstrated greater than $90 \%$ compliance in both groups (results not shown).

\section{Safety measures}

Safety parameters remained unchanged, except for an increase in the GGT-levels $(n=4$ in potassium humate group, $n=2$ in the placebo group). Levels of GGT returned to baseline within 2 weeks of discontinuation of therapy.

\section{Adverse events}

No serious adverse events were noted. Patients receiving potassium humate experienced black stools $(n=3)$ and headache $(n=2)$, while patients on placebo complained of headache $(n=2)$, nausea $(n=2)$, diarrhoea $(n=2)$, flatulence $(n=1)$ and loss of libido $(\mathrm{n}=1)$. 


\section{DISCUSSION}

This is the first study to demonstrate the safety profile of potassium humate, at a daily dose of $1.8 \mathrm{~g}$ in humans, confirming a preclinical toxicity study done in rats [10]. Patients were selected with unilaterally symptomatic idiopathic OA of the knee. All haematology and biochemical parameters stayed within normal ranges during the study. Potassium humate treatment caused a reduction in inflammation in these patients as seen in a reduction in hs-CRP levels as well as clinically significant improvement of patients on the treatment drug compared to the placebo group.

Mechanistic studies done by Joone et al on oxihumate, a watersoluble humate obtained through a wet oxidation of bituminous coal [17], decreases the expression of complement receptor 3 (CR3) by phorbol-12-myristate-13-acetate (PMA) stimulated human neutrophils as well as the adhesion of these cells to a baby hamster kidney cell line expressing intracellular adhesion molecule-1 (ICAM-1) [18], possibly contributing to its antiinflammatory effects. An in vitro finding has recently been documented [19] indicating that brown coal derived potassium humate inhibits the activation of both the classical and alternative pathways of complement activation. It was also shown, in the same study, that potassium humate inhibits the release of the inflammatory related cytokines TNF- $\alpha$, IL-1 $\beta$ and IL-6 in vitro. In the case of arthritis complement activation of both these pathways have been associated with the presence of auto-antibodies against joint components such as type II collagen [20]. Controlling complement activation with monoclonal anti-C5 antibodies has been proven to be effective in decreasing inflammation in animal models of experimental lupus erythematosus, rheumatoid arthritis and septic shock [21]. Regarding the blocking of cytokines, it has been shown that Anakinra, an interleukin-1 (IL-1) receptor antagonist, can be used successfully in the treatment of inflammation and bone destruction in rheumatoid arthritis [22]. Further mechanistic studies need to be done to confirm the mechanism of action of potassium humate.

Studies with bigger sample sizes than this one will have to be done in order to determine whether the results can be replicated. A washout period of more than one week is also recommended.

The identification of a naturally occurring compound that is safe and effective in reducing inflammation in an autoimmune disease such as OA of the knee, merits further evaluation in the treatment 
of patients suffering from other inflammatory arthropathies such as rheumatoid arthritis.

\section{ABBREVIATIONS}

$\mathrm{OA} \quad=$ osteoarthritis

CRP $\quad=$ C-reactive protein

NSAIDs $=$ non-steroidal anti-inflammatory drugs

WOMAC $^{\mathrm{TM}}=$ Western Ontario and McMaster Universities

Osteoarthritis Index questionnaire)

VAS = visual analogue scale

ICAM-1 = intracellular adhesion molecule-1

MCID = minimal clinically important difference

\section{ACKNOWLEDGEMENTS}

This research was supported by Unique Health Trust and a grant from the South African National Research Foundation (NRF).

The corresponding author, Dr C.E.J. van Rensburg, acts as consultant for the company. 


\section{References}

[1] Dawson, J.; Lintel L. Epidemiology of hip and knee pain and its impact on overall health status in older adults. Rheumatology, 2004, 43, 497-504.

[2] Zhang, W.; Jones, A.; Doherty M. Does paracetamol (acetaminophen) reduce the pain of osteoarthritis?: a meta-analysis of randomised controlled trials. Ann. Rheum. Dis., 2004, 63, 901-7.

[3] Joshi, G.P.; White, P.F. Management of acute and postoperative pain. Curr. Opin. Anaesthesiol., 2001; 14, 417-21.

[4] Solomon, D.H. Selective cyclooxygenase 2 inhibitors and cardiovascular events. Arthritis \& Rheumatism., 2005, 52, 196878 .

[5] Rahme, E.; Nedjar H. Risks and benefits of COX-2 inhibitors vs non-selective NSAIDs: does their cardiovascular risk exceed their gastrointestinal benefit? A retrospective cohort study. Rheumatology, 2007, 46: 436-8.

[6] Bonnet, C.S.; Walsh D.A. Osteoarthritis, angiogenesis and inflammation. Rheumatology, 2005, 44, 7-16.

[7] Stürmer, T.; Brenner, H.; Koenig, W.; Günther, K-P. Severity and extent of osteoarthritis and low grade inflammation as assessed by high sensitivity C-reactive protein. Ann. Rheum Dis., 2004, 63, 200-2005.

[8] Visser, S.A.; Some biological effects of humic acids in the rat. Act.a Biol. Med. Germ. Band., 1973, 31, 569-581.

[9] Kleinschmidt, J. Moortherapie bei rheumatischen Erkrankungen; in Moortherapie-Grundlagen und Anwendungen. Wien-Berlin, Ueberreuter, Flaig, W.; Goecke, C.; Kauffels, W., Eds.; 216-224. Wien Berlin: Ueber, 1988.

[10] Van Rensburg, C.E.J.; Snyman, J.R.; Mokoele, T.; Cromarty, A.D. Brown coal derived humate inhibits contact hypersensitivity; an efficacy, toxicity and teragenicity study in rats. Inflammation, 2007, 30, 148-52.

[11] Naude P.J.W.; Cromarty A.D.; Van Rensburg, C.E.J.; Potassium humate inhibits carrageenan-induced paw oedema and a graft-versus-host reaction in rats. Inflammopharmacol, 2010, 18, 339. 
[12] Bellamy, N. Pain assessment in osteoarthritis: experience with the WOMAC osteoarthritis index: Semin. Arthritis Rheum., 1989, 18, 14-7.

[13] Garratt, A.M.; Ruta D.A.; Abdalla M.I.; Buckingham J.K.; Russell I.T. The SF36 health survey questionnaire: an outcome measure suitable for routine use within the NHS? B.M.J., 1993, 306, 1440-4.

[14] Altman, R.; Asch, E.; Bloch, D.; Bole, G.; Borenstein, D.; Brandt, K.; et al: The American College of Rheumatology criteria for the classification and reporting of osteoarthritis of the knee. Arthritis. Rheum., 1986, 29,1039-49.

[15] Ledingham, J.; Regan, M. Factors affecting radiographic progression of knee osteoarthritis. Annals of the Rheumatic Diseases, 1995, 54, 53-8

[16] Vignon, E. Radiographic issues in imaging the progression of hip and knee osteoarthritis. J. Rheumatol. Suppl., 2004, 70, 36-44.

[17] Bergh. J.J.; Cronje, I.J.; Dekker, J.; Dekker, T.G.; Gerritsma, L.M.; Mienie, L.J. Non-catalytic oxidation of waterslurried coal with oxygen: identification of fulvic acids and acute toxicity. Fuel, 1997, 76, 149-54.

[18] Jooné, G.K.; Rensburg, C.E.J. An in vitro investigation of the anti-inflammatory properties of potassium humate.

Inflammation, 2004, 28, 169-74.

[19] Van Rensburg C.E.J.; Naude, P.J.W. Potassium humate inhubits the production of inflammatory cytokines and complement activation in vitro. Inflammation, 2009, 32, 270-6.

[20] Hietala, M.A.; Nandakumar, K.S.; Persson, L.; Fahlen, S.; Holmdahl, R.; Pekna, M. Complement activation by both classical and alternative pathways is critical for the effector phase of arthritis. Eur. J. Immunol., 2004, 34,1208-16.

[21] Kirschfink M. Controlling the complement system in inflammation. Immunopharmacology, 1997, 38, 51-62.

[22] Imran Iqbal I., Fleischm R. Treatment of osteoarthritis with anakinra. Curr. Rheumatol. Rep., 9, 31-35. 
- 14 - 